\title{
SCIENCE INSTRUMENTATION FOR THE STUDENT NITRIC OXIDE EXPLORER
}

\author{
Scott M. Bailey, Richard A. Kohnert, Robert L. McFeeters, Aimee M. Merkel, \\ Erica M. Rodgers, Stanley C. Solomon, Stanley D. Straight, James E. Vian, \\ Thomas N. Woods \\ Laboratory for Atmospheric and Space Physics \\ University of Colorado \\ Boulder CO, 80309-0590 \\ Phone: $303-492-0827$ \\ Fax: 303-492-6444 \\ Email: Bailey@zodiac.colorado.edu
}

\begin{abstract}
The Student Nitric Oxide Explorer (SNOE) is a small satellite to be designed built and operated at the University of Colorado under the Student Explorer Demonstration Initiative (STEDI) from the Universities Space Research Association. The goal of the STEDI program is to demonstrate that low cost satellite missions can be done with large student involvement. The primary science goals of SNOE are to measure thermospheric nitric oxide (NO) and its variability over the lifetime of the mission. SNOE will also monitor the solar irradiance at soft $\mathrm{X}$-ray wavelengths and the auroral energy deposition at high latitudes. Three science instruments are required to achieve the simultaneous measurements: an ultraviolet spectrograph for NO; a solar soft X-ray photometer; and a far ultraviolet photometer for studying the aurora. The instruments are designed to represent a minimum impact on the spacecraft, particularly in terms of data storage and interactions with the command and data handling system. The focus of this paper is the outline of the design of the science instruments and the trade studies which were made. In particular, we discuss why these instruments are well suited for smaller, lower cost satellite missions.
\end{abstract}

\section{Introduction}

The Student Explorer Demonstration Initiative (STEDI) is a program funded by the Universities
Space Research Association (USRA). The goal of STEDI is to demonstrate the feasibility of low cost satellite missions which incorporate large student involvement in the design, fabrication, and operational phases. Two missions have been funded: the Student Nitric Oxide Explorer (SNOE, "snowy") and the Tomographic Experiment using Radiative Recombinative Ionospheric EUV and Radio Sources (TERRIERS, Boston University, D. M. Cotton, PI). Each mission is funded at a level of 4.3 million dollars for two years of design and fabrication and one year of mission operations. The cost of the launch vehicle is not included in this amount.

SNOE is a small spinning spacecraft with a $550 \mathrm{~km}$ orbit and nominal lifetime of one year. The mission science goals are to measure thermospheric nitric oxide (NO) and its variability. SNOE also measures the solar irradiance at soft X-ray wavelengths and the auroral energy deposition at high latitudes. Solar soft $\mathrm{X}$-rays and auroral energy are thought to produce the large variability in NO observed with previous experiments such as the Solar Mesosphere Explorer (SME; Barth et al., 1988; Siskind et al., 1990). Three science instruments are required to achieve the simultaneous measurements of NO, solar soft x-rays, and auroral energy. These are an ultraviolet (UV) spectrometer for NO, a solar soft X-ray photometer, and a far ultraviolet photometer for studying the aurora. 
In order to meet the financial constraints of the STEDI program while accomplishing the science goals, the instrumentation must be designed to have a minimum impact on the spacecraft. Data rates must be kept to a minimum so that lower cost communications systems may be utilized. On SNOE, data is only stored for the portion of the spin during which relevant measurements are made. The output of each of the channels is electronically the same, minimizing the complexity of the interactions with the spacecraft microprocessor. In order to meet design cost limitations, instruments with heritage from other satellite missions and sounding rocket experiments are used. In the following sections, the design of the SNOE science instruments are described.

\section{Mission Overview}

SNOE is scheduled to be launched on a PEGASUS in March of 1997. The SNOE spacecraft is spin

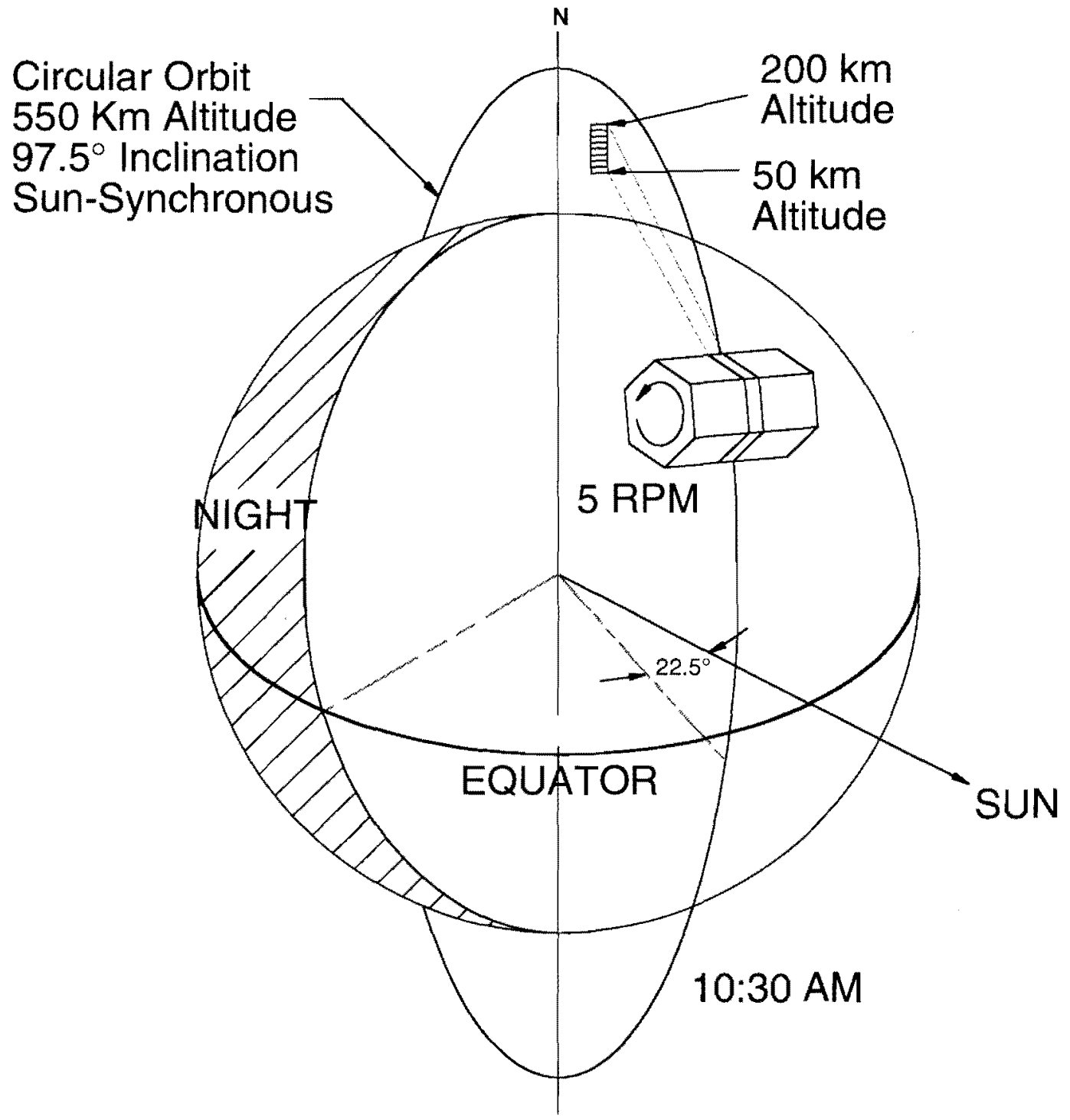

Figure 1. Schematic of SNOE in orbit. 
stabilized with the spin axis normal to the orbital plane. The sun-synchronous orbit is circular at $550 \pm 50 \mathrm{~km}$ altitude with $97.5^{\circ}$. A 10:30-22:30 orbit is selected to meet the science and design requirements. The design requirements are that the solar panels receive sufficient illumination, but that the orbit is sufficiently far from the noon-midnight plane that it will not precess into it during the mission lifetime. Precession can occur due to launch vehicle injection errors. An orbit in the noon-midnight plane is undesirable as it places the sun into the fields of view of the two airglow instruments for part of each spin. The sun is much brighter than the airglow and would saturate the detectors. A program lifetime in orbit of one year is anticipated. For the target altitude range, actual satellite lifetime should be longer, which provides extra launch margin. Requirements for comprehensive mission success are measurements for a period of 81 days (three solar rotations) of nitric oxide altitude profiles, auroral energy flux along the satellite orbit, and solar X-ray emissions. A detailed description of the mission is provided by Solomon et al. (this issue). Figure 1 shows a schematic of SNOE in orbit.

\section{Instrument Descriptions}

The science instrumentation for the SNOE mission consists of: (1) an ultraviolet spectrometer to measure nitric oxide density, (2) an auroral photometer to measure the flux of energetic electrons entering the Earth's atmosphere at high latitude, and (3) a solar soft X-ray photometer to measure the solar irradiance at wavelengths from 2 to 28 $\mathrm{nm}$. The specifications of the individual instruments are now given.

\section{Ultraviolet Spectrometer}

The primary function of the ultraviolet spectrometer (UVS) is to measure the densities of nitric oxide between the altitudes of 50 and $200 \mathrm{~km}$ in the terrestrial upper atmosphere by observing the NO $(1,0)$ and $(0,1)$ gamma bands. The UVS design was developed under the SME program and was successfully flown on SME. Figure 2 shows an optical layout and perspective drawing of the UVS. It consists of an Ebert-Fastie spectrometer, an off-axis telescope, and two Hamamatsu phototube detectors. The spectrometer has a focal length of $125 \mathrm{~mm}$ and uses a $3600 \mathrm{l} / \mathrm{mm}$ mechanically ruled plane grating which produces a dispersion of $1.86 \mathrm{~nm} / \mathrm{mm}$ at the detectors. The phototubes each have fused silica windows and a cesium telluride photocathode. The telescope is an off-axis parabola with a $250 \mathrm{~mm}$ focal length and is used to image the spectrometer slit on the Earth's limb. The combination of the spectrometer and the detectors produces a spacing of $22 \mathrm{~nm}$ between the two channels and the exit slits will be sized to give each detector a $3.5 \mathrm{~nm}$ bandpass. The grating in the spectrometer will be set to place the $(1,0)$ gamma band $(215 \mathrm{~nm})$ on one detector and the $(0,1)$ gamma band $(237 \mathrm{~nm})$ on the other detector. Both channels have a sensitivity of -36 counts/second per Rayleigh/Angstrom.

The UVS is mounted with its optical axis perpendicular to the spin axis of the spacecraft. Its telescope images the entrance slit of the spectrometer on the Earth's limb with the long axis of the slit parallel to the horizon. The image of the slit on the limb is $7 \mathrm{~km}$ high, which determines the fundamental altitude resolution of the instrument. Figure 1 shows the pointing of the UVS in orbit. The integration time of the UVS is set to 2.7 milliseconds for $2 \mathrm{x}$ spatial oversampling. Once the spacecraft has achieved orbit, the UVS high voltage will be turned on and each channel will produce a continuous stream of data. To minimize requirements on the spacecraft, data will only be stored for the downward limb scan. Allowing for some overscan, this produces 50 samples per spin from each channel. The storage operation will be initiated by a signal derived from the 

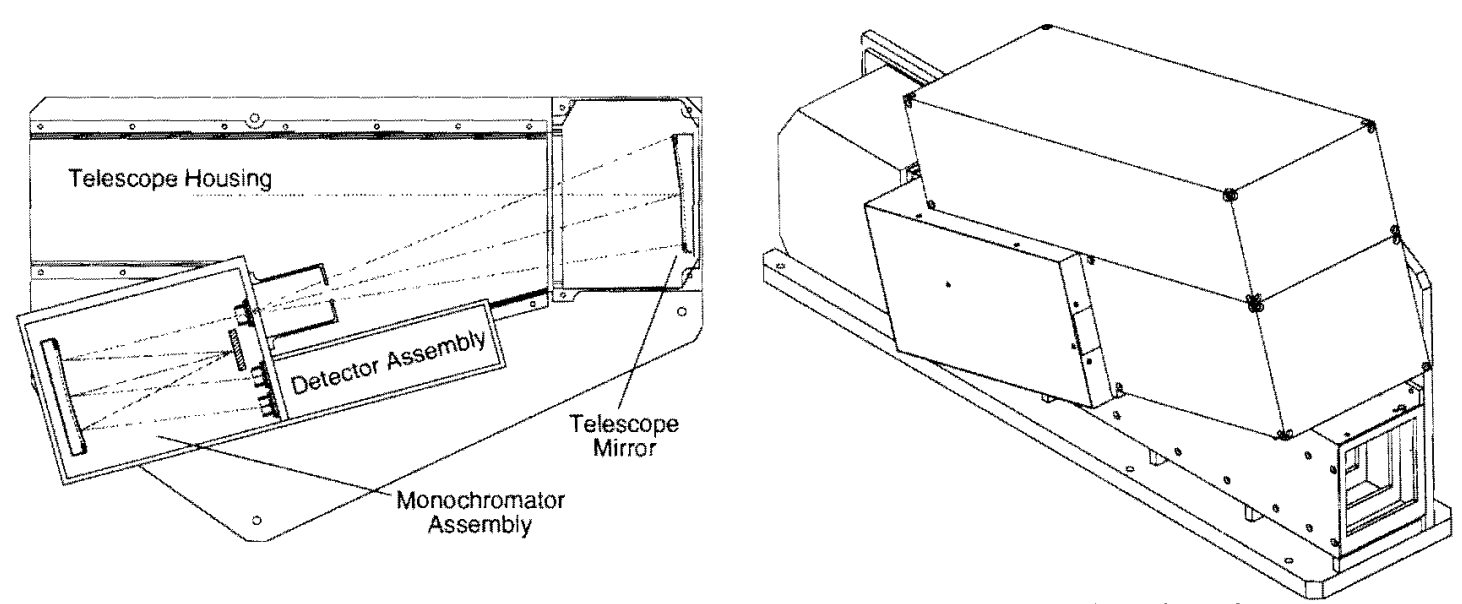

Figure 2. Ultraviolet Spectrometer layout and perspective drawing.

horizon crossing indicator in the ADCS. The data are stored in a buffer which will be periodically emptied, time-tagged, and stored by the spacecraft microprocessor. The UVS electronics are identical to the auroral photometer electronics; a function block diagram appropriate to both of these instruments is shown in Figure 3.

- NO densities can be determined through inversion of the altitude profiles of the observed NO airglow features. Of the two features observed, only the $(1,0)$ band is attenuated as it travels through the atmosphere. The attenuation is due to self absorption by NO molecules along the path. The ratio of the brightnesses of the attenuated and unattenuated emission is dependent upon the abundance of NO. The relationship between this ratio and the column density of NO has been determined and can therefore be used in remote sensing of NO (Eparvier, 1991). This technique will be employed by SNOE. The use of emission ratios rather than absolute brightnesses provides



improved accuracy because ratios do not depend on the absolute calibration of the instrument. The need for only two emissions allows the use of simple detectors rather than the more expensive and intricate imaging detectors frequently used.

\section{Auroral Photometer}

The auroral photometer (AP) is a two-channel broad-band instrument that will be used to determine the energy deposited in the upper atmosphere by energetic auroral electrons. It is a copy of airglow photometers developed by LASP and flown on OGO-5 and -6 in the late 1960 's. A perspective drawing of the AP is shown in Figure 3. The channels (A and B) consist of two Hamamatsu phototube detectors, a UV window/filter for each channel, and a field of view limiter for each channel. Both channels have circular fields of view, $11^{\circ}$ fullcone. The detectors are identical

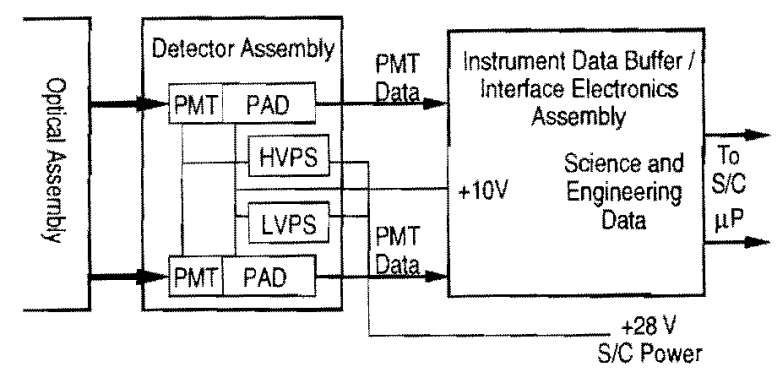

Figure3. Auroral photometer prospective drawing and functional block diagram. 
phototubes with magnesium fluoride $\left(\mathrm{MgF}_{2}\right)$ windows and cesium iodide (CsI) photocathodes. Channel $\mathrm{A}$ has a calcium fluoride $\left(\mathrm{CaF}_{2}\right)$ filter placed in front of the detector and channel $\mathrm{B}$ has a barium fluoride $\left(\mathrm{BaF}_{2}\right)$ filter. The combination of the CsI photocathode and the $\mathrm{CaF}_{2}$ filter produces a bandpass from 125 to $180 \mathrm{~nm}$ for channel $\mathrm{A}$, allowing a combined measurement of the LBH bands, the OI doublet at $135.6 \mathrm{~nm}$, and the OI triplet at $130.4 \mathrm{~nm}$. Channel $B$ has a 135 to $180 \mathrm{~nm}$ bandpass, providing a measurement of the LBH bands and the Or doublet at $135.6 \mathrm{~nm}$ with the exclusion of the OI triplet at $130.4 \mathrm{~nm}$. The sensitivity of channel $A$ at $130.4 \mathrm{~nm}$ is $\sim 3.5$ counts/second/Rayleigh and the sensitivity of channel B at $135.6 \mathrm{~nm}$ is $\sim 14$ counts/second/Rayleigh. The AP and UVS photomultiplier electronics are identical, resulting in significant economies in fabrication and operation.

As with the UVS, the AP is mounted with its optical axis perpendicular to the spacecraft spin axis. The AP produces continuous data but at a much lower rate than the UVS. Only the downward-looking $180^{\circ}$ of each spin (limb-to-limb nadir scan) will be stored. The integration time for each channel is set to 183 milliseconds which provides 32 samples per channel per spin. Data from the $\mathrm{AP}$ are stored in its buffer, which is periodically emptied by the spacecraft microprocessor. A block diagram of the logic electronics is shown in Figure 2.

The pass band of channel $B$ is dominated by the $\mathrm{N}_{2} \mathrm{LBH}$ bands, the brightness of these airglow features is directly proportional to the energy deposited into the atmosphere (Bailey, 1995). Channel $\mathrm{A}$ is dominated by the $\mathrm{O}$ feature at $130.4 \mathrm{~nm}$. The ratio of $\mathrm{Or}$ and $\mathrm{N}_{2}$ emissions is a function of the characteristic energy of the impinging auroral electrons (Strickland et al., 1983). Thus, the simple 2 channel photometer system is capable of describing the energy input to the atmosphere.

\section{$\underline{\text { Solar X-Ray Photometer }}$}

The solar X-ray photometer (SXP) measures the solar irradiance at wavelengths from 2 to $31 \mathrm{~nm}$. Each photometer channel contains a silicon photodiode; wavelength selection is accomplished by thin metallic films deposited directly onto the diode surface. Five photodiodes are flown, Table 1 lists the different coatings and their thicknesses. Coatings are selected so that overlapping bandpasses can be used to isolate key parts of the solar soft X-ray and hard EUV (or "XUV") spectrum at low resolution, including the $2-10 \mathrm{~nm}$ irradiance.

The fields of view are $\sim 70^{\circ}$ fullcone to obtain a solar measurement once per spin during the day. The SXP is mounted to point $22.5^{\circ}$ from the spacecraft spin axis. This geometry places the sun at the center of the field of view once during each dayside spin for a $10: 30$ orbit.

Each photodiode is followed by a current amplifier and a voltage-tofrequency converter, resulting in a

Table 1. Solar X-ray photometer channels

\begin{tabular}{|cccccc|}
\hline Channel & $\begin{array}{c}\text { Metallic } \\
\text { Coating }\end{array}$ & $\begin{array}{c}\text { Thickness } \\
(\mathrm{nm})\end{array}$ & $\begin{array}{c}\text { Passband } \\
(\mathrm{nm})\end{array}$ & $\begin{array}{c}\text { Active Area } \\
\left(\mathrm{cm}^{2}\right)\end{array}$ & $\begin{array}{c}\text { Peak Sensitivity } \\
\text { (electrons/photon at } \lambda)\end{array}$ \\
\hline 1 & $\mathrm{Ti}$ & 250 & $2-5$ & 0.1 & 40 at $2 \mathrm{~nm}$ \\
2 & $\mathrm{Sn}$ & 300 & $2-10$ & 0.1 & 15 at $5 \mathrm{~nm}$ \\
3 & $\mathrm{Zr} / \mathrm{Ti} / \mathrm{C}$ & $200 / 5 / 50$ & $10-17$ & 0.1 & 15 at $10 \mathrm{~nm}$ \\
4 & $\mathrm{Al} / \mathrm{C} / \mathrm{Sc}$ & $200 / 50 / 50$ & $17-28$ & 0.1 & 5 at $17 \mathrm{~nm}$ \\
5 & none & - & UV-Visible & .0001 & 0.7 at $500 \mathrm{~nm}$ \\
\hline
\end{tabular}


sequence of pulses with a frequency proportional to the diode current. These pulses are counted over 62.5 millisecond integration periods by the instrument electronics and stored in a data buffer that is periodically emptied by the spacecraft microprocessor. The use of the voltageto-frequency converter has the advantages of allowing a larger dynamic range in the measurements and providing an output equivelent to that of the phototubes in the other instruments. Instruments of this design have flown on LASP and HAO sounding rockets six times (Woods et al., 1994; Bailey, 1995). A perspective drawing and functional block diagram of the instrument electronics are shown in Figure 4.

Part of the measured current is due to visible-wavelength radiation entering through microscopic flaws in the photodiode coating. To measure these background currents a door mechanism fitted with a fused silica window is included. The fused silica allows all visible light to reach the photodiode but rejects light below $160 \mathrm{~nm}$, thus when the door is closed the signal is completely due to background visible light. The window is opened and closed once in an orbit and the process is repeated every other orbit. The X-ray signal is obtained by subtracting data taken with the window closed from data taken with the window open. Transmission of the window is measured using the channel 5 photodiode which has no coating. The stable visible sunlight will be used to detect any window degradation. Dark currents will be measured by looking at the dark sky.

A small two-axis sun sensor is co-aligned with the SXP to measure the solar incidence angle for the instrument. This measurement is useful because the measured signal will vary as the cosine of this angle. This sensor was developed at LASP for the rocket program and was flight tested in on two sounding rockets; a copy will be fabricated for SNOE.
This instrument is well suited for use on small satellites. The simple output of a photodiode allows for low data rates and simplified control circuitry. The combined use of several photodiodes provides adequate spectral resolution while allowing a lower data rate than a readout detector in a grating spectrograph. The ability to directly deposit the thin film to the surface of the photodiodes is a significant simplification over the use of foil filters which are difficult to handle and tend to develop pinholes after time.

\section{Instrument Logic}

The electrical interfaces to the spacecraft are identical for each instrument. This is possible because the UVS and AP have identical detector systems and because the output of an SXP channel is electrically identical to a PMT. Equivalent circuitry has the advantages of reducing both design and test time and therefore overall cost. Every spin, data storage for each instrument is initiated by a signal from a pair of Horizon Crossing Indicators (HCI).

The instrument logic performs the following functions: interprets command words from the spacecraft microprocessor, initiates data storage based upon signal from $\mathrm{HCI}$, and informs the spacecraft microprocessor that the data buffer is ready to be read. The command word provides the logic interface with integration time, delay time between integration periods, and delay time between $\mathrm{HCI}$ pulse and data storage initiation. For the UVS and AP the delay time between integration periods is typically zero.

The electrical design for the logic interface is based upon Field Programmable Gate Arrays (FPGA). A single FPGA can be used to perform many logic functions and therefore dramatically reduces the number of 

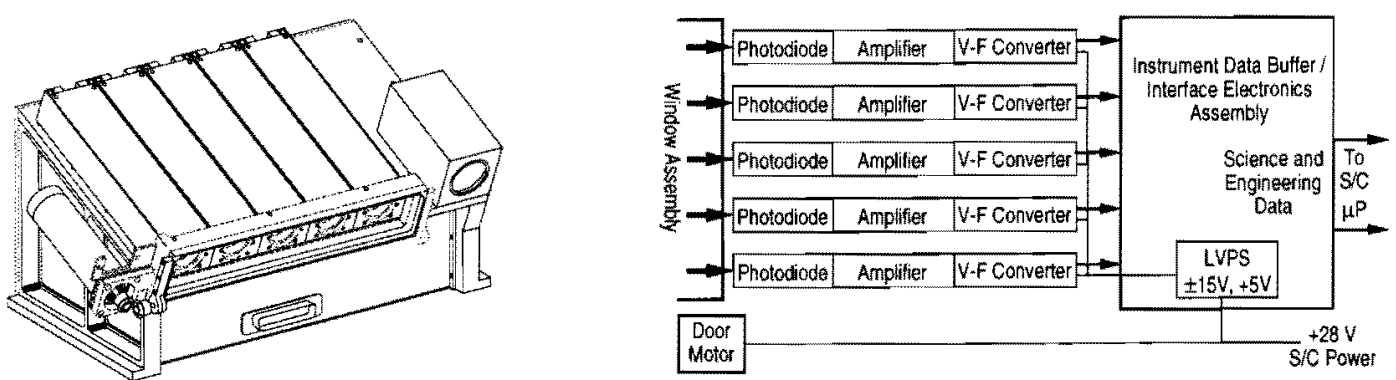

Figure 4. Solar X-ray photometer perspective drawing and functional block diagram.

integrated circuits required. The result is a streamlined design with reduced packaging and lower power requirements.

\section{$\underline{\text { Summary }}$}

The science instruments for the SNOE mission have been described. SNOE is a low cost satellite mission utilizing large student involvement in all phases of the project. In order to accomplish the science goals while meeting the constraints of the STEDI program, relatively simple science instruments with high flight heritage are used. A design which minimizes the impact of the instruments on the spacecraft is crucial. Such a design is achieved on SNOE by using instruments which have identical electrical interfaces. Data rates are kept to a minimum by storing data only during those portions of the spin where useful measurements are made. SNOE will launch in March of 1977 and will be operated in orbit for one year.

\section{$\underline{\text { References }}$}

Bailey, S. M., Response of the Upper Atmosphere to Variations in the Solar Soft X-ray Irradiance, Ph. D. Dissertation, University of Colorado, 1995.

Barth, C. A., W. K. Tobiska, D. E. Siskind, and D. D. Cleary, Solar-Terrestrial Coupling: Low Latitude Thermospheric Nitric Oxide, Geophys. Res. Lett., 15, 92 , 1988.

Eparvier, F. G., Nitric Oxide in the Lower Thermosphere, PhD Thesis, University of Colorado, 1991.

Siskind, D. E., C. A. Barth, and D. D. Cleary, The Possible Effect of Solar Soft X Rays on Thermospheric Nitric Oxide, J. Geophys. Res., 95, 4311, 1990.

Strickland, D. J., J. R. Jasperse, and J. A. Whalen, Dependence of Auroral FUV Emissions of the Incident Electron Spectrum and Neutral Atmosphere, J. Geophys. Res., 88, 8051, 1983.

Woods, T. N., G. J. Rottman, S. M. Bailey and S. C. Solomon, Far Ultraviolet and Extreme Ultraviolet Instrumentation for Measuring the Solar Spectral Irradiance and the Terrestrial Airglow, Optical Engineering, 33, 438, 1994. 


\begin{abstract}
Scott Bailey is a research associate at the Laboratory for Atmospheric and Space Physics (LASP) at the University of Colorado where he received his Ph.D. in 1995. Prior to LASP, he worked as a cooperative education student at the Naval Research Laboratory. Research Interests include experimental studies of the upper atmosphere and atmospheres of the planets and solar variability and its effect on the Earth. At LASP, he has specialized in sounding rocket experiments and is currently focusing on the SNOE mission.
\end{abstract}

